\title{
ATRA improves endothelial dysfunction in atherosclerotic rabbits by decreasing CAV-1 expression and enhancing eNOS activity
}

\author{
YAN WU ${ }^{1,2^{*}}$, XIAOBIAN WANG ${ }^{2 *}$, QING ZHOU $^{2}$, YI WANG $^{2}$, JIALI ZHOU ${ }^{2}$, \\ QIAOLING JIANG ${ }^{2}$, YUAN WANG ${ }^{2}$ and HUAQING ZHU ${ }^{2}$ \\ ${ }^{1}$ Reproductive Medicine Center, 105 Hospital of People's Liberation Army, Hefei, Anhui 230031; \\ ${ }^{2}$ Laboratory of Molecular Biology, Department of Biochemistry, Anhui Medical University, Hefei, Anhui 230032, P.R. China
}

Received December 28, 2015; Accepted December 9, 2016

DOI: $10.3892 / \mathrm{mmr} .2018 .8647$

\begin{abstract}
The aim of the present study was to explore the protective effects and possible mechanisms of all-trans-retinoic acid (ATRA) against atherosclerosis (AS). Rabbits were randomly allocated for standard or high-fat diet with or without ATRA. After 12 weeks, the aortic rings of the rabbits were removed. Endothelium-dependent relaxation (EDR) induced by acetylcholine and non-endothelium-dependent relaxation induced by sodium nitroprusside in the thoracic aorta were evaluated. NO level and eNOS activity were measured according to the protocol of NO and eNOS ELISA kits. The permeability and morphology of the arterial walls were identified by immunofluorescence and $\mathrm{H} \& \mathrm{E}$ staining respectively. The expression of caveolin-1 (CAV-1) and occludin was analyzed using western blotting and immunohistochemistry. The EDR function was significantly reduced in the AS rabbits compared with the normal group, however it was elevated following treatment with ATRA. The eNOS activity and NO level were reduced in the AS group, however were notably increased following oral administration of ATRA. There was an enhancement of endothelial permeability in the AS group compared with the normal group, which decreased following ATRA treatment. Western blot analysis and immunohistochemical analysis identified an increase in occludin expression after treatment with ATRA, in contrast to CAV-1 expression under the same conditions. ATRA is able to ameliorate high-fat-induced AS in rabbits, which is mediated through the activation of eNOS and downregulating CAV-1 expression.
\end{abstract}

Correspondence to: Professor Huaqing Zhu or Dr Yuan Wang, Laboratory of Molecular Biology, Department of Biochemistry, Anhui Medical University, 81 Meishan Road, Hefei, Anhui 230032, P.R. China

E-mail: aydzhq@126.com

E-mail: wangyuan@ahmu.edu.cn

*Contributed equally

Key words: all-trans-retinoic acid, atherosclerosis, caveolin-1, occludin, endothelial dependent relaxation

\section{Introduction}

Atherosclerosis (AS) is a complex multifactorial disease characterized by the concentration of large scale lipids, inflammatory cells and fibrous elements. Evidence is accumulating to suggest that damage to vascular endothelial cells initiates AS (1). Endothelial cells form a permeable barrier, which maintains the internal environment by manufacturing and excreting various cytokines and by regulating cellular cholesterol, lipid homeostasis and inflammation in the vascular wall (2). NO is a potent vasodilator factor and signal regulator in endothelial cells, and is induced by eNOS through catalyzing L-arginine. The NO/eNOS system is crucial in endothelium-dependent diastolic function and damage to endothelial function generally always follows a decrease in eNOS activity (3). Caveolae consist of caveolin, cholesterin and sphingomyelin, and are thus termed from their flask-shaped, invaginated structures observed in the cytoplasmic membrane. CAV-1 is an essential structural protein for the formation of caveolae, and is expressed in endothelial cells, macrophages and smooth muscle cells (4). It has been demonstrated that CAV-1 may serve as a target site for AS by restraining eNOS activity by combining with eNOS (5-7).

All-trans retinoic acid (ATRA), a derivative of vitamin A, is involved in inducing cell differentiation, reducing inflammation and suppressing cell proliferation and metastasis (8). Previous studies $(9,10)$ have demonstrated that ATRA is effective in delaying the process of AS, although the exact mechanism remains to be elucidated. The majority of studies exploring the function of ATRA focus on vascular smooth muscle (VSMC), however, few (11-14) have demonstrated that ATRA regulates the activity of eNOS to affect NO concentration in endothelial cells.

The present study attempted to investigate the therapeutic effect of ATRA in AS rabbits, in addition to the potential mechanisms of ATRA-attenuated AS.

\section{Materials and methods}

Reagents. ATRA was purchased from Sigma-Aldrich (Merck Millipore, Darmstadt, Germany). Anti-occludin (cat. no. sc-8144), anti-CAV-1 (cat. no. sc-70516) and anti- $\beta$-actin (cat. no. sc-47778) were obtained from Santa Cruz Biotechnology, Inc. (Dallas, TX, USA). All secondary 
antibodies were purchased from EMD Millipore (Billerica, MA, USA). HRP-conjugated secondary antibodies, including rabbit-anti-goat $(1: 1,000$, cat. no. AP106P), goat-anti-mouse (1:1,000, cat. no. AP127P) and goat-anti-mouse (1:2,000, cat. no. AP127P) were purchased from EMD Millipore (Billerica, MA, USA). Histostain-plus kits and 3,3'-diaminobenzidine (DAB) horseradish peroxidase (HRP) color development kits were obtained from OriGene Technologies, Inc. (Beijing, China). eNOS and NO kits were purchased from Nanjing Jiancheng Bioengineering Institute (Nanjing, China). Sulfo-NHS-LC-biotin was obtained from Pierce (Thermo Fisher Scientific, Inc., Waltham, MA, USA).

Animal experimental procedures. The present study was approved by the Ethics Committee of Anhui Medical University (Hefei, China). All rabbits were bred humanely in compliance with the 'Principles of Laboratory Animal Care' formulated by the USA National Society for Medical Research and the Guide for the Care and Use of Laboratory Animals. A total of 36 4-month-old and weight $1.8 \pm 0.2 \mathrm{~kg}$ male New Zealand white rabbits were obtained from Nanjing Anlimo Technology Co., Ltd. (Nanjing, China), and maintained in individual cages under moderate temperature and a 12-h light/dark cycle, with ad libitum access to food and clean water. Following a 7-day acclimation period, they were randomly divided into the control group $(n=10)$, an AS group (AS group, $n=10)$ and an ATRA treatment group (ATRA group, $n=10$ ). Rabbits in the control group were fed a normal diet (150 g/day), the AS group were fed a high-cholesterol diet (1\% cholesterol and 5\% lard, $150 \mathrm{~g} /$ day), and the ATRA group received a high-cholesterol diet and $10 \mathrm{mg} / \mathrm{kg} /$ day ATRA. The control and the AS groups received the same volume of medium by gavage. All the rabbits were euthanized at the end of week 12 .

Tissue collection. Animals were anaesthetized by $3 \%$ pentobarbital sodium (Sigma-Aldrich; Merck KGaA; $1 \mathrm{ml} / \mathrm{kg}$ ) and then sacrificed by rapid exsanguination. The aortic arch, the thoracic and abdominal aorta, and the arteria iliaca were removed. The aortic arch was cut longitudinally and fixed in $4 \%$ paraformaldehyde for pathological and immunohistochemical assays. The upper section of the abdominal aorta was placed in a dish containing ice-cold Krebs solution (composition in mmol/l: $\mathrm{NaCl}, 120 ; \mathrm{KCl}, 4.7 ; \mathrm{KH}_{2} \mathrm{PO}_{4}, 1.18 ; \mathrm{CaCl}_{2}$, 2.25; $\mathrm{NaHCO}_{3}, 24.5 ; \mathrm{MgSO}_{4} \cdot 7 \mathrm{H}_{2} \mathrm{O}, 1.2$; glucose, 11.1; EDTA, 0.03 ) and continuously aerated with $95 \% \mathrm{O}_{2}$ and $5 \% \mathrm{CO}_{2}$; the samples were then cut into rings ( $3 \mathrm{~mm}$ in length) for the measurement of isometric contractile tension. The remaining arteries were frozen in liquid nitrogen and stored at $-80^{\circ} \mathrm{C}$ for western blotting.

Measurement of isometric contractile tension. Individual aortic rings were vertically suspended between two stainless steel wire hooks in a jacketed organ bath containing $25 \mathrm{ml}$ Krebs solution (as described above), which was replaced at 15 -min intervals. The bathing solution was aerated continuously with a mixture of $95 \% \mathrm{O}_{2}$ and $5 \% \mathrm{CO}_{2}$ at $37^{\circ} \mathrm{C}$. Isometric contractile tension was continuously recorded using a BL-420F experimental system of biological function (Chengdu Tai Meng Science and Technology Co., Ltd., Chengdu, China). Resting tension was increased stepwise to reach a final tension of $2 \mathrm{~g}$ that was applied to the aortic rings; then, they were equilibrated for $45 \mathrm{~min}$. Following equilibration, rings were precontracted with $1 \times 10^{-6} \mathrm{~mol} / 1$ phenylephrine (Phe) and, once a stable contraction plateau was obtained, $1 \times 10^{-9}-1 \times 10^{-4} \mathrm{~mol} / \mathrm{l}$ acetylcholine (ACh) or $1 \times 10^{-9}-1 \times 10^{-4} \mathrm{~mol} / 1$ sodium nitroprusside (SNP) was cumulatively added to the organ bath until a maximal vasodilator response was achieved. Cumulative vasodilator response data were expressed as the percentage of relaxation relative to the Phe-induced precontraction.

Immunofluorescence. Arteries were unfolded on freezing optimal cutting temperature compound and treated with Sulfo-NHS-LC-biotin for $30 \mathrm{~min}$, then preserved at $-80^{\circ} \mathrm{C}$. The frozen sections were washed in PBS 3 times $(10 \mathrm{~min}$ each), blocked with $5 \%$ non-fat milk overnight at $4^{\circ} \mathrm{C}$, then incubated with Rhodamin (1:20) for $2 \mathrm{~h}$ at $4^{\circ} \mathrm{C}$, then washed three times prior to mounting and coverslipping. The sections were observed using a Leica immunofluorescence microscope (Leica Microsystems GmbH, Wetzlar, Germany).

Immunohistochemistry. The expression levels of CAV-1 and occludin in the aortas were measured by immunohistochemistry. The sections were dewaxed in xylene, rehydrated in graded ethanol solutions and subjected to antigen retrieval in citrate-buffered solution at $95^{\circ} \mathrm{C}$ for $15 \mathrm{~min}$. Endogenous peroxidase activity was blocked by $3 \%$ hydrogen peroxide. The specimens were blocked by normal goat serum and incubated with the anti-CAV-1 or anti-occludin overnight. After being washed in PBS, the slides were incubated with streptavidin-biotin horseradish peroxidase complex after biotin-conjugated secondary antibody. Then the sections were treated with DAB for 5 min and, after thorough washing, were mounted on slide glasses with Resinene (Guo Yao Chemical Reagent Co., Ltd., Shanghai, China).

Western blot analysis. The aortas were washed with PBS for 3 times, and lysed in RIPA buffer (Tris-HCl, $\mathrm{pH} \mathrm{7.14,}$ $150 \mathrm{mmol} / 1 \mathrm{NaCl}, 1 \mathrm{mmol} / 1$ EDTA, $1 \%$ Triton X-100, 0.1\% SDS, $5 \mathrm{mg} / \mathrm{ml}$ leupeptin and $1 \mathrm{mmol} / \mathrm{l} \mathrm{PMSF})$. The lysates were centrifuged at $14,000 \mathrm{x}$ g for $30 \mathrm{~min}$ at $4^{\circ} \mathrm{C}$. The protein concentration of each sample was measured with Micro-BCA Protein Assay Reagent kit (Beyotime Institute of Biotechnology, Haimen, China). Protein extracts were blended with SDS sample buffer and boiled for $8 \mathrm{~min}$, separated through $12 \%$ SDS-PAGE, and then transferred onto polyvinylidene difluoride membranes. The membranes were blocked with 5\% fat-free milk in PBST (PBS, $0.1 \%$ Tween-20) for $2 \mathrm{~h}$ at room temperature, and then incubated overnight with anti-CAV-1 (1:500), anti-occludin (1:500) or anti- $\beta$-actin $(1: 1,000)$ at $4^{\circ} \mathrm{C}$, followed by the appropriate HRP-conjugated secondary antibody with CAV-1, occludin or $\beta$-actin, and detected with enhanced chemiluminescence (Beyotime Institute of Biotechnology). Protein bands were visualized by exposing the blots to Kodak X-ray film (Kodak, Rochester, NY, USA).

Statistical analysis. Statistical analyses were conducted using SPSS, version 19.0 (IBM SPSS, Armonk, NY, USA). All data are expressed as the mean \pm standard deviation. One-way analysis of variance was used to evaluate the statistical significance 
Table I. Endothelium-dependent relaxation of thoracic aorta produced by $\mathrm{ACh}(\%, \mathrm{n}=6$, mean $\pm \mathrm{SD})$.

\begin{tabular}{lrrr}
\hline ACh $(\mathrm{mol} / \mathrm{l})$ & \multicolumn{1}{c}{ Normal } & \multicolumn{1}{c}{ Model } & \multicolumn{1}{c}{ ATRA } \\
\hline $10^{-9}$ & $4.83 \pm 0.26$ & $1.65 \pm 0.19^{\mathrm{a}}$ & $1.99 \pm 0.19^{\mathrm{a}, \mathrm{b}}$ \\
$10^{-8}$ & $14.66 \pm 1.23$ & $3.98 \pm 0.45^{\mathrm{a}}$ & $9.48 \pm 1.31^{\mathrm{a}, \mathrm{b}}$ \\
$10^{-7}$ & $52.52 \pm 1.92$ & $22.79 \pm 2.98^{\mathrm{a}}$ & $34.56 \pm 2.12^{\mathrm{a}, \mathrm{b}}$ \\
$10^{-6}$ & $77.45 \pm 2.97$ & $44.06 \pm 5.76^{\mathrm{a}}$ & $55.99 \pm 3.67^{\mathrm{a}, \mathrm{b}}$ \\
$10^{-5}$ & $84.52 \pm 3.19$ & $50.88 \pm 1.63^{\mathrm{a}}$ & $68.51 \pm 3.14^{\mathrm{a}, \mathrm{b}}$ \\
$10^{-4}$ & $95.68 \pm 3.57$ & $53.34 \pm 2.11^{\mathrm{a}}$ & $73.98 \pm 3.08^{\mathrm{a}, \mathrm{b}}$ \\
\hline
\end{tabular}

${ }^{\mathrm{a}} \mathrm{P}<0.05$ vs. normal group, ${ }^{\mathrm{b}} \mathrm{P}<0.05$ vs. model group. Values are presented as the percentage of relaxation relative to a phenylephrine precontraction $(1 \mu \mathrm{m})$ and data are expressed as the mean \pm standard deviation ( $\mathrm{n}=6 /$ group). ACh, acetylcholine; ATRA, all-trans-retinoic acid; SD, standard deviation.

Table II. Non-endothelium-dependent relaxation of thoracic aorta produced by $\operatorname{SNP}(\%, n=6$, mean $\pm \mathrm{SD})$.

\begin{tabular}{lrrr}
\hline SNP $(\mathrm{mol} / \mathrm{l})$ & \multicolumn{1}{c}{ Normal } & \multicolumn{1}{c}{ Model } & \multicolumn{1}{c}{ ATRA } \\
\hline $10^{-9}$ & $7.34 \pm 0.33$ & $7.22 \pm 0.59$ & $7.16 \pm 0.37$ \\
$10^{-8}$ & $32.64 \pm 1.03$ & $34.00 \pm 1.80$ & $33.32 \pm 2.34$ \\
$10^{-7}$ & $64.38 \pm 1.96$ & $62.61 \pm 1.88$ & $62.55 \pm 3.57$ \\
$10^{-6}$ & $87.35 \pm 2.62$ & $86.54 \pm 2.72$ & $88.29 \pm 3.95$ \\
$10^{-5}$ & $97.01 \pm 2.28$ & $96.96 \pm 3.97$ & $98.14 \pm 4.60$ \\
$10^{-4}$ & $106.55 \pm 3.93$ & $106.20 \pm 4.22$ & $107.99 \pm 4.68$ \\
\hline
\end{tabular}

Values are presented as percentage of relaxation relative to a phenylephrine precontraction $(1 \mu \mathrm{m})$ and data are expressed as mean \pm standard deviation ( $\mathrm{n}=6$ /group). $\mathrm{SNP}$, sodium nitroprusside; ATRA, all-trans-retinoic acid; SD, standard deviation.

of the differences between multiple groups. $\mathrm{P}<0.05$ was considered to indicate a statistically significant difference.

\section{Results}

ATRA improved endothelium-dependent relaxation (EDR) function in AS rabbits. EDR of thoracic aorta was induced by ACh ranging from $10^{-9}-10^{-4} \mathrm{~mol} / \mathrm{l}$. As presented in Table I, the maximum relaxation induced by $\mathrm{ACh}\left(10^{-9} \mathrm{~mol} / \mathrm{l}\right)$ in the normal group was $95.68 \%$ compared with $53.34 \%$ in the AS group. This suggested that the EDR function in the AS group was seriously impaired compared with the normal group $(\mathrm{P}<0.05)$. Treatment with ATRA markedly ameliorated this damage and restored the relaxation to $73.98 \%$ compared with the AS group, indicating that ATRA makes a contribution to EDR in AS rabbits. There was no notable difference on SNP-induced non-endothelium-dependent relaxation (NEDR) in the thoracic aortic rings (Table II).

ATRA improves the permeability of the arterial wall in AS rabbits. The permeability of endothelial cells was detected by immunofluorescence (surface biotinylation technique).

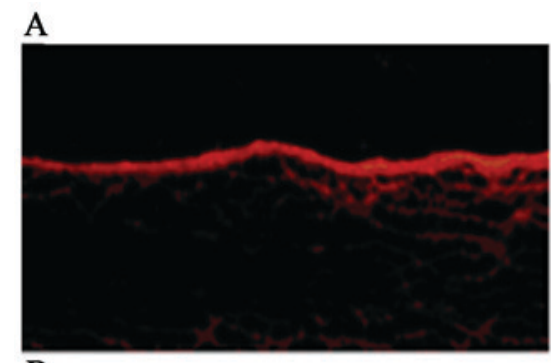

B
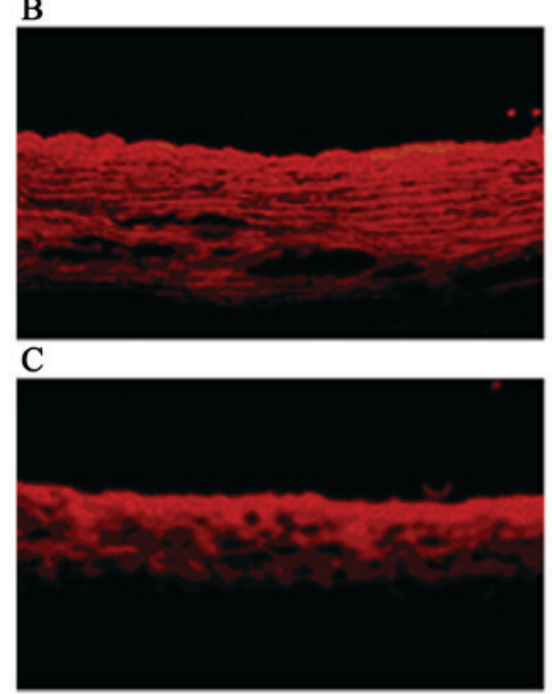

Figure 1. The permeability of the arterial walls detected by immunofluorescence in the three groups (x200). (A) There was a little paracellular leakage of NHS-LC-biotin in the normal arterial wall, however in the (B) model group all the layers of the artery were biotinylated. (C) The leakage of NHS-LC-biotin through the arterial intima was markedly reduced in AS rabbits following treatment with ATRA. AS, atherosclerosis; ATRA, all-trans-retinoic acid.

Concentration profiles of NHS-LC-biotin were acquired according to the radial distance through the media of the arterial wall. There was a little paracellular leakage of NHS-LC-biotin in the normal arterial walls (Fig. 1A) in contrast, all the arterial layers were biotinylated in the AS rabbits (Fig. 1B). As presented in Fig. 1C, leakage of NHS-LC-biotin, which permeates through arterial intima, was reduced markedly in AS rabbits following treatment with ATRA, suggesting that ATRA was able to restore the permeability of damaged endothelial to some extent.

ATRA attenuated CAV-1 protein and enhanced occludin expression level in AS rabbits. The features of the arterial lesions were examined by hematoxylin and eosin (H\&E) staining. The arterial intima was clear and intact in the normal group and the endothelial cell cores were stained and evenly arranged (Figs. 2A and 3A). However broken arterial intima, increased intercellular space, numerous foam cells and fibrous plaques were observed in the AS group (Figs. 2B and $3 \mathrm{~B}$ ). Treatment with ATRA resulted in fewer foam and inflammatory cells, and no fibrous plaques (Figs. 2C and 3C). Immunohistochemical analysis demonstrated that the expression of occludin was lower in the AS rabbits compared with the normal rabbits, while the level of occludin was markedly increased following ATRA treatment (Fig. 2). In addition, western blot analysis gave the similar results for occludin 
$\mathrm{A}_{\mathrm{a}}$

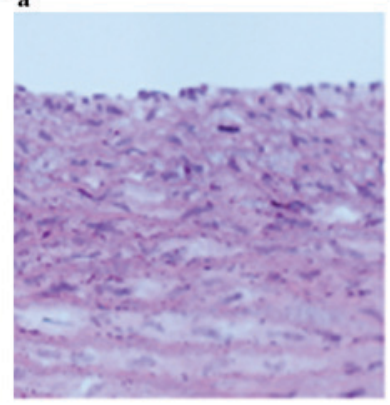

b

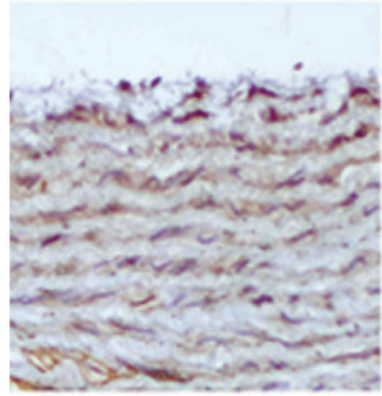

$\mathrm{B}_{\mathrm{a}}$

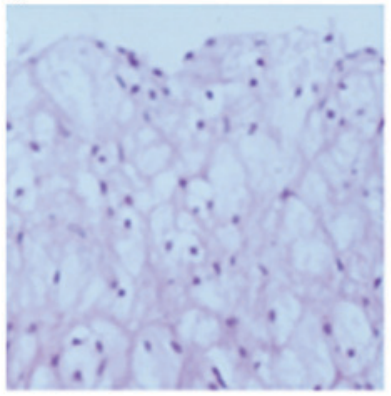

b

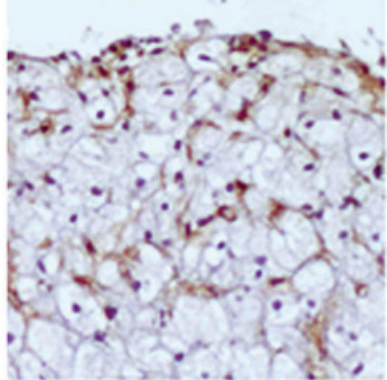

$\mathrm{C}_{\mathrm{a}}$

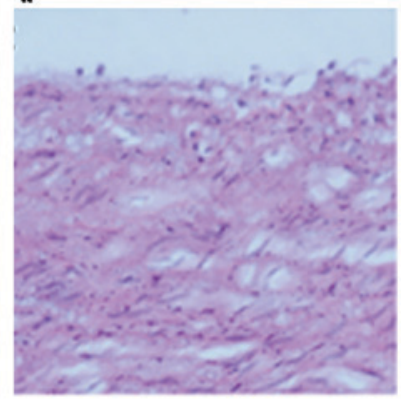

b

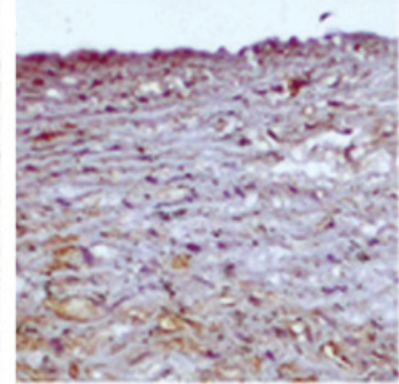

Figure 2. H\&E and immunohistochemical staining of occludin in arterial walls (x200). (Aa) H\&E staining of the arterial walls normal group; the arterial intima was clear and complete. (Ba) The model group; the arterial intima was damaged, and numerous foam cells and fibrous plaque were discovered. (Ca) The ATRA group; fewer foam and inflammatory cells, and no fibrous plaques. Immunohistochemical staining of occludin demonstrated that the staining in (Ab) normal rabbits was higher compared with (Bb) AS rabbits and that it was ( $\mathrm{Cb}$ ) clearly increased following treatment with ATRA. H\&E, hematoxylin and eosin; AS, atherosclerosis; ATRA, all-trans-retinoic acid.

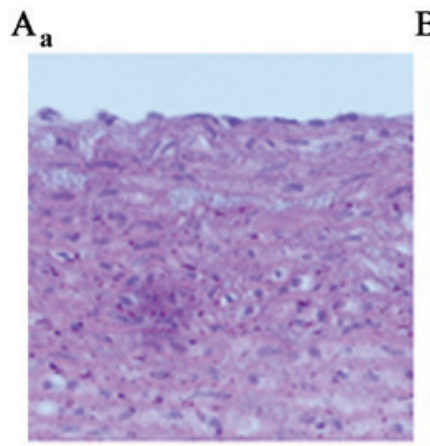

b

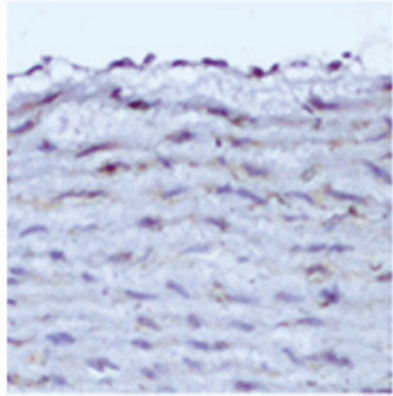

$\mathrm{B}_{\mathrm{a}}$

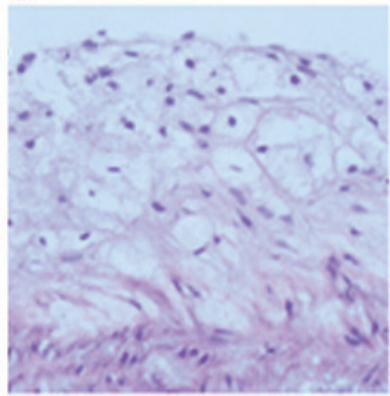

b

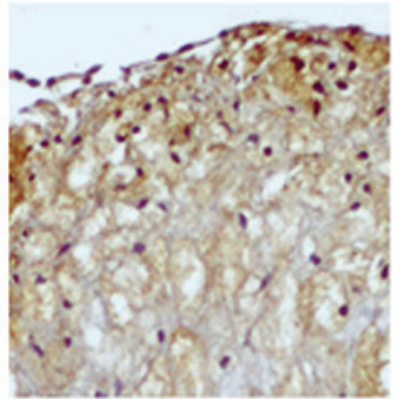

$\mathrm{C}_{\mathrm{a}}$

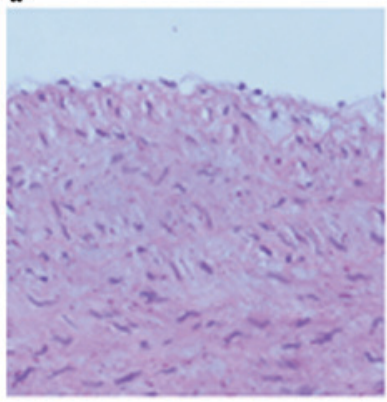

b

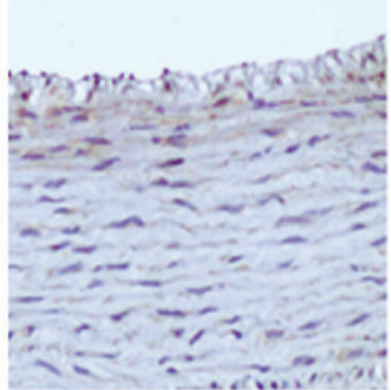

Figure 3. H\&E and immunohistochemical staining of CAV-1 in arterial walls (x200). The result of H\&E staining was the same as Fig. 2 (Aa, Ba and Ca). Immunohistochemical staining of CAV-1 in AS rabbits was higher than normal rabbits, while it was markedly reduced when treated with ATRA (Aa, Ba and $\mathrm{Ca}$ ). H\&E staining of the arterial walls (Aa) normal group (Ba) model group (Ca) ATRA group. Immunohistochemical staining of the arterial walls (Ab) normal group $(\mathrm{Bb})$ model group $(\mathrm{Cb})$ ATRA group. H\&E, hematoxylin and eosin; AS, atherosclerosis; ATRA, all-trans-retinoic acid.

expression (Fig. 4). As demonstrated in Figs. 3 and 5, immunohistochemistry gave the same indications of the expression of CAV-1 as did western blot analysis, unlike occludin under the same conditions.
ATRA increased eNOS activity and NO concentration in AS rabbits. As presented in Table III, eNOS activity and NO concentration of the AS group were markedly decreased compared with the normal group $(\mathrm{P}<0.05)$, nevertheless they 
Table III. Comparison of eNOS activity and NO concentration in three groups $(\mathrm{n}=10, \overline{\mathrm{x}} \pm s)$.

\begin{tabular}{lcc}
\hline Group & $\begin{array}{c}\text { eNOS activity } \\
(\mathrm{U} / \mathrm{mgprot})\end{array}$ & $\begin{array}{c}\text { NO concentration } \\
(\mu \text { mol/gprot })\end{array}$ \\
\hline Normal & $6.53 \pm 0.86$ & $18.56 \pm 9.77$ \\
Model & $0.82 \pm 0.62^{\mathrm{a}}$ & $1.52 \pm 0.42^{\mathrm{a}}$ \\
ATRA & $3.75 \pm 2.57^{\mathrm{a}, \mathrm{b}}$ & $7.72 \pm 2.81^{\mathrm{a}, \mathrm{b}}$ \\
\hline
\end{tabular}

${ }^{\mathrm{a}} \mathrm{P}<0.05$ vs. normal group, ${ }^{\mathrm{b}} \mathrm{P}<0.05$ vs. model group. Data are expressed as the mean \pm standard deviation ( $n=6 /$ group). ATRA, all-trans-retinoic acid.

were notably increased in the AS rabbits following treatment with ATRA.

\section{Discussion}

ATRA is part of a family of signaling molecules that are derived from vitamin A, and emergent studies have concentrated on its antitumor effects $(15,16)$. Now it has been demonstrated that ATRA may serve an important function in AS (17). AS is identified as a common pathological basis of cardio-cerebrovascular disease, which involves a variety of risk factors and etiological mechanisms. Although the etiological factors and pathological mechanisms remain to be fully elucidated, the widely accepted hypothesis postulates that the endothelial damage is regarded as the initiation of AS $(18,19)$. To further elucidate the potential mechanisms of ATRA in AS was the aim of the present study.

Endothelial cells, located between blood and tissue, form a cellular barrier to circulating blood and have an important role in maintaining vascular function, regulating the permeability and balancing the coagulation and fibrinolysis systems $(20,21)$. Injury of the endothelial cells leads to an alteration in EDR in response to ACh. In the present study, the maximal relaxation induced by $\mathrm{ACh}\left(10^{-9} \mathrm{~mol} / \mathrm{l}\right)$ was only $53.34 \%$ in the AS group, suggesting that the EDR function was seriously impaired in the AS rabbits compared with the normal rabbits. After treatment with ATRA, the relaxation was restored to $73.98 \%$, indicating that ATRA makes a contribution to EDR in AS rabbits (Table I). In the present study, experiments on isolated thoracic aorta rings demonstrated that there was no notable difference on SNP-induced NEDR (Table II).

It has been demonstrated that the exchange of substances between the inside and outside of the blood vessel, including signaling molecules and structural proteins, depends on endothelial permeability. That permeability is regulated by the adhesive force maintained by cell-cell junctions and cell-matrix contacts $(22,23)$. The present study demonstrated that the permeability of the arterial wall was increased after the rabbits were provided with a high-fat diet for 12 weeks, while endothelial permeability had recovered following treatment with ATRA (Fig. 1). The results indicated ATRA as a regulator to adjust the damaged endothelial permeability in the AS rabbits.
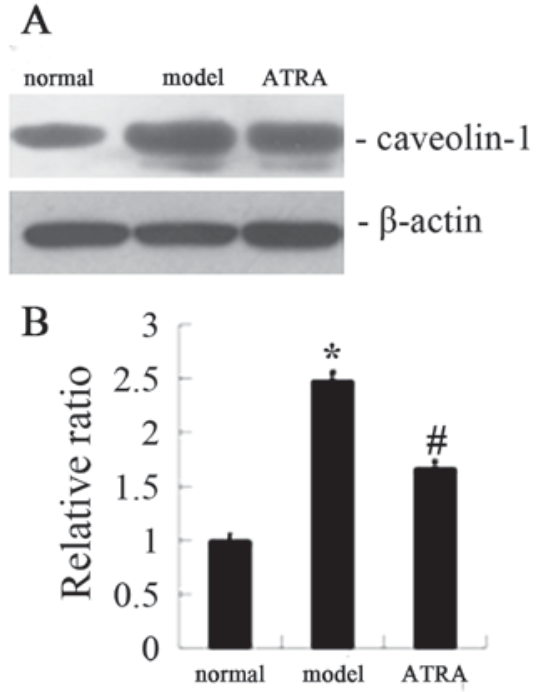

Figure 4. ATRA enhanced the expression of occludin in arterial walls. (A) Western blot analysis demonstrated that the expression of occludin in AS rabbits was the lowest of three groups, while it was enhanced visibly treated with the ATRA group. (B) Densitometric units of the normal group (occludin $/ \beta$-actin). ${ }^{*} \mathrm{P}<0.05$ vs. normal group and ${ }^{*} \mathrm{P}<0.05$ vs. model group. ATRA, all-trans-retinoic acid; AS, atherosclerosis.
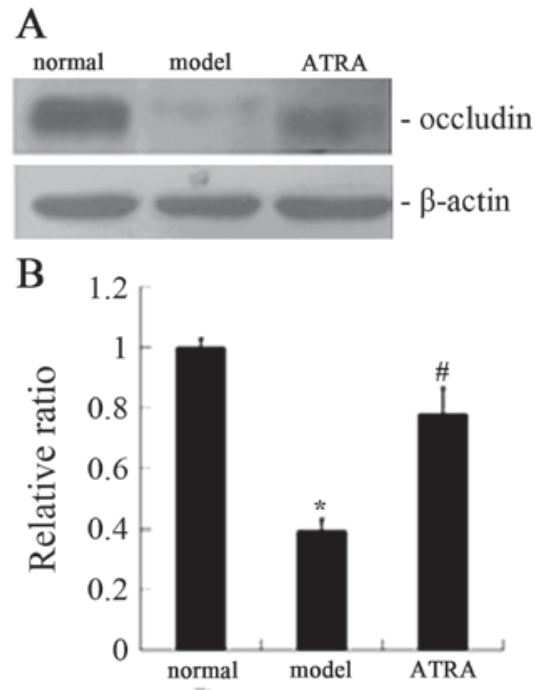

Figure 5. ATRA attenuated the expression of CAV-1 in the arterial wall. The expression of CAV-1 in the AS group was higher than that in normal rabbits, however, the level of CAV-1 was markedly decreased following ATRA treatment. (A) Western blot analysis; (B) densitometric units of the normal group (CAV-1/ $\beta$-actin). ${ }^{*} \mathrm{P}<0.05$ vs. normal group and ${ }^{\#} \mathrm{P}<0.05$ vs. model group. ATRA, all-trans-retinoic acid.

Tight junctions (TJs), which consist of occludin, the claudin protein family and ZO-1, are vital structures generated in epithelial and endothelial cells, regulating the paracellular permeation of ions and macromolecules (24). Acting as an important constituent of TJs, occludin restricts the flow of fluid from the vascular lumen to the intercellular space (25). Although it is disputed that occludin maintains the integrity of TJs, a study demonstrated that TJs may be damaged following a deficiency of occludin (26), causing the migration of hemameba and lipids, the increase of endothelial permeability and ultimately leading to AS formation. The present 
study noted that the expression of occludin was lower in AS rabbits compared with normal rabbits (Fig. $2 \mathrm{Ab}$ and $\mathrm{Bb}$ ) according to immunohistochemical analysis, while occludin expression level was markedly increased after ATRA treatment (Fig. 2Cb). Western blot analysis yielded the same results as immunohistochemical analysis (Fig. 4). These results indicated that ATRA improves the permeability of the aorta intima by upregulating occludin expression.

The results of the present study demonstrated that the anti-AS effects of ATRA were associated with NO, an endothelium-derived relaxing factor that is released by endothelial cells. NO is one of the most important vasoactive compounds in vivo, restraining VSMC migration and proliferation, platelet aggregation and leukocyte adhesion (27). Endothelial dysfunction, a precondition of $\mathrm{AS}$, is always accompanied by the decrease of NO synthesis and release, in addition to NO activity and bioavailability (28). It has been established that ATRA can increase NO concentration via two ways: i) Changing asymmetric dimethylarginine, the inhibitor of eNOS system and ii) enhancing the phosphorylation of eNOS to increase NO formation (29). The present study demonstrated that eNOS activation and NO concentration in the AS group were significantly reduced compared with the normal group, while they were clearly increased after treatment with ATRA (Table III).

The phosphorylation of eNOS at serine 1177 by the phosphoinositide 3-kinase-protein kinase $\mathrm{B}$ pathway is associated with NO release (30), while a previous study (31) demonstrated that CAV-1 is able to inhibit eNOS activity by forming an eNOS-CAV-1 complex in endothelial cells. When cells are stimulated, eNOS is displaced from the eNOS-CAV-1 complex and activated by combining with calmodulin, making a contribution to the increase of NO concentration (32). Here, immunohistochemical analysis indicated that the expression of CAV-1 was increased in AS rabbits compared with normal rabbits (Fig. 3Ab and $\mathrm{Bb}$ ), while the level of $\mathrm{CAV}-1$ was notably decreased following ATRA treatment (Fig. 3Cb). Western blot analysis confirmed the results of immunohistochemical analysis (Fig. 5).

In summary, ATRA has a promising effect in the amelioration of high-fat-induced AS in rabbits, and it may produce its protective effects by activating eNOS, in addition to downregulating CAV-1 expression. However, the exact and detailed mechanism of ATRA function remains to be elucidated.

\section{Acknowledgements}

The present study was supported by the National Natural Science Foundation of China (grant nos. 81570419 and 81270372), Key Project of Chinese Ministry of Education (grant no. 212077) and Grants for Scientific Research of BSKY (grant nos. XJ201107 and XJ2008015) from Anhui Medical University.

\section{References}

1. Fernández-Hernando C, Yu J, Dávalos A, Prendergast J and Sessa WC: Endothelial-specific overexpression of caveolin-1 accelerates atherosclerosis in apolipoprotein E-Deficient mice. Am J Pathol 177: 998-1003, 2010.

2. Sima AV, Stancu CS and Simionescu M: Vascular endothelium in atherosclerosis. Cell Tissue Res 335: 191-203, 2009.
3. Liu D, He Z, Wu L and Fang Y: Effects of induction/inhibition of endogenous heme oxygenase-1 on lipid metabolism, endothelial function, and atherosclerosis in rabbits on a high fat diet. J Pharmacol Sci 118: 14-24, 2012.

4. Zulli A, Buxton BF, Black MJ, Ming Z, Cameron A and Hare DL: The immunoquantification of caveolin-1 and eNOS in human and rabbit diseased blood vessels. J Histochem Cytochem 54: 151-159, 2006.

5. Xu Y, Buikema H, van Gilst WH and Henning RH: Caveolae and endothelial dysfunction: Filling the caves in cardiovascular disease. Eur J Pharmacol 585: 256-260, 2008.

6. Lee MH, Chen SJ, Tsao CM and Wu CC: Perivascular adipose tissue inhibits endothelial function of rat aortas via caveolin-1. PLoS One 9: e99947. 2014.

7. Luo DX, Cheng J, Xiong Y, Li J, Xia C, Xu C, Wang C, Zhu B, $\mathrm{Hu} \mathrm{Z}$ and Liao DF: Static pressure drives proliferation of vascular smooth muscle cells via caveolin-1/ERK1/2 pathway. Biochem Biophys Res Commun 391: 1693-1697, 2010.

8. Siddikuzzaman, Guruvayoorappan C and Berlin Grace VM: All trans retinoic acid and cancer. Immunopharmacol Immunotoxicol 33: 241-249, 2011.

9. Axel DI, Frigge A, Dittmann J, Runge H, Spyridopoulos I, Riessen R, Viebahn R and Karsch KR: All-trans retinoic acid regulates proliferation, migration, differentiation, and extracellular matrix turnover of human arterial smooth muscle cells. Cardiovasc Res 49: 851-862, 2001.

10. Herdeg C, Oberhoff M, Baumbach A, Schroeder S, Leitritz M, Blattner A, Siegel-Axel DI, Meisner C and Karsch KR: effects of local all-trans-retinoic acid delivery on experimental atherosclerosis in the rabbit carotid artery. Cardiovasc Res 57: 544-553, 2003.

11. Uruno A, Sugawara A, Kanatsuka H, Kagechika H, Saito A, Sato K, Kudo M, Takeuchi K and Ito S: Upregulation of nitric oxide production in vascular endothelial cells by all-trans retinoic acid through the phosphoinositide 3-kinase/Akt pathway. Circulation 112: 727-736, 2005.

12. Cho DH, Choi YJ, Jo SA, Nam JH, Jung SC and Jo I: Retinoic acid decreases nitric oxide production in endothelial cells: A role of phosphorylation of endothelial nitric oxide synthase at Ser(1179). Biochem Biophys Res Commu 326: 703-710, 2005

13. Drolet MC, Plante E, Battistini B, Couet J and Arsenault M: Early endothelial dysfunction in cholesterol-fed rabbits: A non-invasive in vivo ultrasound study. Cardiovasc Ultrasound 2: 10, 2004.

14. Yang AL, Yeh CK, Su CT, Lo CW, Lin KL and Lee SD: Aerobic exercise acutely improves insulin and insulin-like growth factor-1-mediated vasorelaxation in hypertensive rats. Exp Physiol 95: 622-629, 2010.

15. Bushue N and Wan YJ: Retinoid pathway and cancer therapeutics. Adv Drug Deliv Rev 62: 1285-1298, 2010.

16. Ye Xf, Wu Q, Liu S, Lin Xf, Zhang B, Wu Jf, Cai Jh, Zhang Mq and $\mathrm{Su} \mathrm{Wj}$ : Distinct role and functional mode of TR3 and RARalpha in mediating ATRA-induced signalling pathway in breast and gastric cancer cells. Int J Biochem Cell Biol 36: 98-113, 2004.

17. Zhou B, Pan Y, Hu Z, Wang X, Han J, Zhou Q, Zhai Z and Wang Y: All-trans-retinoic acid ameliorated high fat diet-induced atherosclerosis in rabbits by inhibiting platelet activation and inflammation. J Biomed Biotechnol 2012: 259693, 2012.

18. Hansson GK, Robertson AK and Söderberg-Nauclér C: Inflammation and atherosclerosis. Annu Rev Pathol 1: 297-329, 2006.

19. Sitia S, Tomasoni L, Atzeni F, Ambrosio G, Cordiano C, Catapano A, Tramontana S, Perticone F, Naccarato P, Camici P, et al: From endothelial dysfunction to atherosclerosis. Autoimmun Rev 9: 830-834, 2010.

20. Simionescu M and Antohe F: Functional ultrastructure of the vascular endothelium: Changes in various pathologies. Handb Exp Pharmacol: 41-69, 2006.

21. Mehta D and Malik AB: Signaling mechanisms regulating endothelial permeability. Physiol Rev 86: 279-367, 2006.

22. Yuan SY: Protein kinase signaling in the modulation of microvascular permeability. Vascul Pharmacol 39: 213-223, 2002.

23. Shepro D: The american microcirculatory society landis award lecture: Endothelial cells, inflammatory edema, and the microvascular barrier: Comments by a 'free radical'. Microvasc Res 35: 246-264, 1988.

24. Suzuki H, Nishizawa T, Tani K, Yamazaki Y, Tamura A, Ishitani R, Dohmae N, Tsukita S, Nureki O and Fujiyoshi Y: Crystal structure of a claudin provides insight into the architecture of tight junctions. Science 344: 304-307, 2014. 
25. McKenzie JA and Ridley AJ: Roles of Rho/ROCK and MLCK in TNF-alpha-induced changes in endothelial morphology and permeability. J Cell Physiol 213: 221-228, 2007.

26. Zhao J, Hao J, Fei X, Wang X, Hou Y and Deng C: Isoflurane inhibits occludin expression via up-regulation of hypoxia-inducible factor $1 \alpha$. Brain Res 1562: 1-10, 2014.

27. Dias RG, Negrão CE and Krieger MH: Nitric oxide and the cardiovascular system: Cell activation, vascular reactivity and genetic variant. Arq Bras Cardiol 96: 68-75, 2011.

28. Verma S, Buchanan MR and Anderson TJ: Endothelial function testing as a biomarker of vascular disease. Circulation 108 2054-2059, 2003.

29. Achan V, Tran CT, Arrigoni F, Whitley GS, Leiper JM and Vallance P: All-trans-retinoic acid increases nitric oxide synthesis by endothelial cells: A role for the induction of dimethylarginine dimethylaminohydrolase. Circ Res 90: 764-769, 2002.
30. Figueroa XF, González DR, Puebla M, Acevedo JP, Rojas-Libano D, Durán WN and Boric MP: Coordinated endothelial nitric oxide synthase activation by translocation and phosphorylation determines flow-induced nitric oxide production in resistance vessels. J Vasc Res 50: 498-511, 2013.

31. Trane AE, Pavlov D, Sharma A, Saqib U, Lau K, van Petegem F, Minshall RD, Roman LJ and Bernatchez PN: Deciphering the binding of caveolin-1 to client protein endothelial nitric-oxide synthase (eNOS): Scaffolding subdomain identification, interaction modeling, and biological significance. J Biol Chem 289: 13273-13283, 2014.

32. Du YH and Chen AF: A new role for caveolin-1: Regulation of guanosine triphosphate cyclohydrolase I and tetrahydrobiopterin in endothelial cells. Hypertension 53: 115-117, 2009. 